\title{
An Efficient Game Form for Multi-rate Multicast Service Provisioning
}

\author{
Ali Kakhbod and Demosthenis Teneketzis Fellow, IEEE
}

\begin{abstract}
We consider the decentralized bandwidth/rate allocation problem in multi-rate multicast service provisioning with strategic users. We demonstrate that such a situation is the combination of a market problem and a public goods problem. We present a mechanism/game form which possesses the following properties when the users' utilities are concave: (1) It implements in Nash equilibria the solution of the corresponding centralized rate allocation problem in multi-rate multicast service provisioning. (2) It is individually rational. (3) It is budget-balanced at all Nash equilibria of the game induced by the mechanism/game form as well as at all off equilibrium messages/strategies that result in feasible allocations.
\end{abstract}

Index Terms- Multi-rate Multicast Service Provisioning, Strategic users, Mechanism design, Markets, Public goods, Nash equilibrium.

\section{INTRODUCTION}

\section{A. Motivation and Challenges}

Multicasting provides an efficient method of transmitting data in real time applications from one source to many users. The source sends one copy of a message to its users and this copy is replicated only at the branching points of a multicast tree. Real life examples of such multicast applications are audio/video broadcasting, teleconferencing, distributed databases, financial information, electronic newspapers, weather maps and experimental data. Conventional multicast studies the problem in which the rate received by all the users of the same multicast group is constant. The inherent problem with such a formulation is that a constant rate will overwhelm the slow receivers while starving the fast ones. multi-rate multicast transmissions can be used to address this problem by allowing a receiver to obtain data at a rate that satisfies its requirements. Single-rate and multi-rate multicast problems have been extensively studied. A survey of the existing literature on multicast and its comparison with the results of this paper appears later in this section.

All existing literature on multi-rate multicast assumes nonstrategic users. As we explain in section I-B below, the nature of the problem suggests that strategic behavior may be beneficial to the users. Strategic behavior results in new challenges (conceptual and technical) in multi-rate multicast. In this paper we study, within the context of mechanism design, the decentralized rate allocation problem in multirate multicast with strategic users. We formulate the problem, propose a mechanism for rate allocation, analyze the properties of the proposed mechanism, compare our results with the

Ali Kakhbod and Demosthenis Teneketzis are with the department of EECS, University of Michigan, Ann Arbor, MI - 48109, USA. (email: \{akakhbod, teneket\}@umich.edu). Their work was supported in part by NSF Grant CCR-0325571 and by NSF Grant CCF-1111061. existing literature on multi-rate multicast, and discuss open problems.

\section{B. Why is strategic behavior justified?}

Strategic behavior in multi-rate multicast can be justified as follows. The literature on multi-rate multicast with nonstrategic users reveals that the problem has characteristics of the free-rider problem. That is, at any network link, a member of a multicast group is charged only if it requests the maximum rate/bandwidth within the group at that link. As a result of this feature of the problem, users are incentivized to misrepresent their demand for bandwidth; by slightly reducing its demand, a user can increase its overall utility because it slightly reduces its own satisfaction from the quality of service it receives, but pays considerably less tax. Thus, strategic behavior may result in higher overall utility for a user than non-strategic behavior.

\section{Contribution of the paper}

The main contributions of this paper are:

1) The formulation of the multi-rate multicast service provisioning problem in wired networks with arbitrary topology and strategic users.

2) The discovery of a decentralized rate allocation mechanism for multi-rate multicast service provisioning in networks with arbitrary/general topology and strategic users, which possesses the following properties.

(P1) The mechanism implements the solution of the centralized multi-rate multicast service provisioning problem in Nash equilibria. That is, the allocation corresponding to each Nash equilibria (NE) of the game induced by the mechanism is a globally optimal solution of the corresponding centralized multi-rate multicast service provisioning problem.

(P2) The mechanism is individually rational, that is, the network users/users voluntarily participate in the rate allocation process.

(P3) The mechanism is budget-balanced ${ }^{1}$ at all feasible allocations, that is, at all the allocations that correspond to NE messages/strategies as well as at all the allocations that correspond to off-equilibrium messages/strategies.

The results of this paper are also a contribution to the theory of mechanism design. In Section II we show that the multirate multicast problem with strategic users is the combination of a market and a public goods problem with strategic users. Such problems have not been previously investigated within the context of mechanism design.

\footnotetext{
${ }^{1}$ In a budget-balanced mechanism the sum of the taxes paid by a subset of users is equal to the sum of subsidies received by the rest of the users.
} 


\section{Comparison with related work}

Within the context of single rate and multi-rate multicast problems, studies have addressed issues of bandwidth/rate allocation [1]-[10], [16], [17], routing [8], [11]-[13] and reliability [14], [15]. Most of the literature on rate allocation is done via the notion of fairness [1], [2], [6], [7], [9]. The authors of [1] develop a unified framework for diverse fairness objectives via the notion of fair allocation of utilities. A more general approach to rate allocation is via utility maximization. Utility maximization is more general because rate allocation with the fairness property is utility maximizing when the utility has a special form. The authors of [10], [16] and [17] investigated multi-rate multicast problems with a utility maximization objective. In all the aforementioned papers, it is assumed the agents/users are not strategic, that $i s$, they are price-takers who are willing to follow/obey the rules of the resource allocation mechanism.

In contrast to all the above papers, our paper considers the multi-rate multicast problems with strategic users, that is, users which are self-utility maximizers, and do not necessarily obey the rules of the resource allocation mechanism, but have to be incentivized/induced to follow them. To the best of our knowledge, our paper is the first to present a mechanism possessing properties (P1)-(P3) for the multi-rate multicast service provisioning problem with strategic users.

\section{E. Organization of the paper}

The rest of the paper is organized as follows. In section II we formulate the multi-rate multicast service provisioning problem with strategic users. In section III we describe the allocation mechanism/game form we propose for the solution of the multi-rate multicast service provisioning problem. In section IV we analyze the properties of the proposed mechanism. We conclude in section V.

\section{The MUlTi-RATE MUltiCAST PROBlem With STRATEGIC NETWORK USERS, PROBLEM FORMULATION}

In this section we present the formulation of the multirate multicast problem in wired communication networks with strategic users. We proceed as follows, In section III-B we formulate the centralized multi-rate multicast problem the solution of which we want to implement in Nash equilibria. In section III-C we formulate the decentralized multi-rate multicast problem with strategic network users; We state our assumptions, our objective and provide an interpretation of the equilibrium concept (Nash equilibrium) in which we want to implement the solution of the centralized problem of section III-B.

\section{A. The centralized problem}

We consider a wired network with $N$ disjoint groups of strategic users; we denote the set of groups by $\mathcal{N}=$ $\left\{G_{1}, G_{2}, \cdots, G_{N}\right\}$. The network topology, the capacity of the network links, and the routes assigned to users' services are fixed and given. We denote user $j$ in group $G_{i}$ by $\left(j, G_{i}\right)$. The utility function of user $\left(j, G_{i}\right), G_{i} \in \mathcal{N}$, has the form

$$
V_{\left(j, G_{i}\right)}\left(x_{\left(j, G_{i}\right)}, t_{\left(j, G_{i}\right)}\right)=U_{\left(j, G_{i}\right)}\left(x_{\left(j, G_{i}\right)}\right)-t_{\left(j, G_{i}\right)} .
$$

The term $U_{\left(j, G_{i}\right)}\left(x_{\left(j, G_{i}\right)}\right)$ expresses user $\left(j, G_{i}\right)$ 's satisfaction from the service $x_{\left(j, G_{i}\right)}$ it receives. The term $t_{\left(j, G_{i}\right)}$ represents the tax (money) user $\left(j, G_{i}\right)$ pays for the services it receives. We assume that $U_{\left(j, G_{i}\right)}$ is a concave and increasing function of the service $x_{\left(j, G_{i}\right)}$ user $\left(j, G_{i}\right)$ receives, and $t_{\left(j, G_{i}\right)} \in \mathbb{R}$. When $t_{\left(j, G_{i}\right)}>0$ user $\left(j, G_{i}\right)$ pays money for the services it receives; this money is paid to other network users. When $t_{\left(j, G_{i}\right)}<0$ user $\left(j, G_{i}\right)$ receives money from other users. Overall, the amount of money paid by some of the network users must be equal to the amount of money received by the rest of the users so that $\sum_{G_{i} \in \mathcal{N}} \sum_{j \in G_{i}} t_{\left(j, G_{i}\right)}=0$.

Denote: by $\mathbf{L}$ the set of links of the network; by $c_{l}$ the capacity of link $l$; by $\mathcal{R}_{\left(j, G_{i}\right)}$ the set of links $l, l \in \mathbf{L}$, that form the route of user $\left(j, G_{i}\right)$, (as pointed out above each user's route is fixed); by $G_{i}(l)$ the set of users in $G_{i}$ who use link $l$, i.e., $G_{i}(l)=\left\{j: j \in G_{i}\right.$ and $\left.l \in \mathcal{R}_{\left(j, G_{i}\right)}\right\}$; by $x_{G_{i}}(l)$ the maximum amount of bandwidth requested by group $G_{i}$ at link $l$, i.e., $x_{G_{i}}(l):=\max _{j \in G_{i}(l)}\left\{x_{\left(j, G_{i}\right)}\right\}$; by $G_{i}{ }^{\max }(l)$ the set of users in $G_{i}$ using link $l$ and request $x_{G_{i}}(l)$ amount of bandwidth, i.e., $G_{i}{ }^{\max }(l):=\left\{\left(j, G_{i}\right): x_{\left(j, G_{i}\right)}=x_{G_{i}}(l)\right\}$; by $\left(j, G_{i}^{\max }(l)\right)$ a user in $G_{i}^{\max }(l)$; by $\mathbf{L}_{G_{i}}$ the set links used by users in group $G_{i}$, i.e., $\mathbf{L}_{G_{i}}:=\left\{l: \exists\left(j, G_{i}\right)\right.$ s.t. $\left.l \in \mathcal{R}_{\left(j, G_{i}\right)}\right\}$; by $\mathcal{R}_{\left(j, G_{i}\right)}^{\max }$ the set of links $l, l \in \mathcal{R}_{\left(j, G_{i}\right)}$, such that $x_{\left(j, G_{i}\right)}=x_{G_{i}}(l)$, i.e. $\mathcal{R}_{\left(j, G_{i}\right)}^{\max }=\left\{l: l \in \mathcal{R}_{\left(j, G_{i}\right)}\right.$ s.t. $\left.\left(j, G_{i}\right)=\left(j, G_{i}^{\max }(l)\right)\right\}$; by $Q_{l}$ the set of groups that include at least one user using link $l$, i.e., $Q_{l}:=\left\{G_{i}: l \in \mathbf{L}_{G_{i}}\right\}$.

We assume that a central authority (the network manager) has access to all of the above information. The objective of this authority is to solve the following centralized optimization problem that we call Max.0.

$$
\max _{x, t} \sum_{G_{i} \in \mathcal{N}} \sum_{j \in G_{i}}\left[U_{\left(j, G_{i}\right)}\left(x_{\left(j, G_{i}\right)}\right)-t_{\left(j, G_{i}\right)}\right]
$$

subject to

$$
\begin{aligned}
& \sum_{G_{i} \in Q_{l}} \max _{j \in G_{i}(l)} x_{\left(j, G_{i}\right)} \leq c_{l}, \quad \forall l \in \mathbf{L}, \\
& \sum_{G_{i} \in \mathcal{N}} \sum_{j \in G_{i}} t_{\left(j, G_{i}\right)}=0, \\
& x_{\left(j, G_{i}\right)} \geq 0, \quad \forall j \in G_{i}, G_{i} \in \mathcal{N},
\end{aligned}
$$

where $(x, t)=\left(x_{\left(j, G_{i}\right)}, t_{\left(j, G_{i}\right)}, j \in G_{i}, G_{i} \in \mathcal{N}\right)$. The inequalities in (2) express the capacity constraints that must be satisfied at each network link. The equality in (3) expresses the fact that the budget must be balanced, i.e., the total amount of money paid by some of the users must be equal to the amount of money received by the rest of the users. The inequalities in (4) express the fact that the users' received rates $x_{\left(j, G_{i}\right)}, G_{i} \in \mathcal{N}$, must be nonnegative. Every $(x, t)$ that 
satisfies (2)-(4) is called a feasible allocation/solution.

Problem Max.0 is equivalent to problem Max.1 below,

$$
\max _{x} \sum_{G_{i} \in \mathcal{N}} \sum_{j \in G_{i}} U_{\left(j, G_{i}\right)}\left(x_{\left(j, G_{i}\right)}\right)
$$

subject to

$$
\begin{aligned}
& \sum_{G_{i} \in Q_{l}} \sum_{\left(j, G_{i}\right) \in G_{i}(l)} x_{\left(j, G_{i}\right)} \leq c_{l}, \quad \forall j \in G_{i}(l), l \in \mathbf{L} \\
& x_{\left(j, G_{i}\right)} \geq 0, \forall j \in G_{i}, \quad G_{i} \in \mathcal{N},
\end{aligned}
$$

in the following sense. The set of inequalities in (5) and (6) result in the same domain of solutions $x$ as the set of inequalities in (2) and (4). Thus, any optimal solution $\left(x_{\left(j, G_{i}\right)}, j \in G_{i}, G_{i} \in \mathcal{N}\right)$ of problem Max.1 along with any $t=\left\{t_{\left(j, G_{i}\right)}, j \in G_{i}, G_{i} \in \mathcal{N}\right\}$ such that $\sum_{G_{i} \in \mathcal{N}} \sum_{j \in G_{i}} t_{\left(j, G_{i}\right)}=0$ is also an optimal solution $\left(x_{\left(j, G_{i}\right)}^{*}, t_{\left(j, G_{i}\right)}^{*}, j \in G_{i}, G_{i} \in \mathcal{N}\right)$ of Max.o. We will refer to Max.1 as the centralized multi-rate multicast problem.

Let $E(l)$ be the set of inequalities defined by (5) for link $l$. Evey element of $E(l)$ is denoted by $e(l)(e(l) \in E(l))$. Define $E\left(l,\left(j, G_{i}\right)\right) \subseteq E(l)$ by

$$
E\left(l,\left(j, G_{i}\right)\right):=\left\{e(l) \subseteq E(l): x_{\left(j, G_{i}\right)} \text { appears in } e(l)\right\} .
$$

Let $\mathcal{U}$ denote the set of functions

$$
U: \mathbb{R}_{+} \cup\{0\} \rightarrow \mathbb{R}_{+} \cup\{0\}
$$

where $U$ is concave and increasing, and $\mathbb{R}_{+}$denotes the set of non-negative real numbers. Let $\mathbf{T}$ denote the set of all possible network topologies, network resources and user routes. Consider problem Max.1 for all possible realizations

$$
\times_{G_{i} \in \mathcal{N}} \times_{j \in G_{i}} U_{\left(j, G_{i}\right)} \times T \in \mathcal{U}^{\sum_{G_{i} \in \mathcal{N}}\left|G_{i}\right|} \times \mathbf{T},
$$

of the users' utilities, the network topology, its resources and the users' routes. Then the solution of Max.1 for each $(\mathbf{U}, T) \in \mathcal{U}^{\sum_{G_{i} \in \mathcal{N}}\left|G_{i}\right|} \times \mathbf{T}$ defines a map

$$
\Gamma: \mathcal{U}^{\sum_{G_{i} \in \mathcal{N}}\left|G_{i}\right|} \times \mathbf{T} \rightarrow \mathcal{A},
$$

where $\mathcal{A} \in \mathbb{R}_{+}^{\sum_{G_{i} \in \mathcal{N}}\left|G_{i}\right|}$ is the set of all possible rate/bandwidth allocations to the network's users. We call $\Gamma$ the solution of the centralized problem.

\section{B. The decentralized problem with strategic users}

We consider the network model of the previous section with the following assumptions on its information structure.

(A1) Each user knows only his own utility; this utility is his own private information. Each user also knows the function space $\mathcal{U}$ to which the utilities of all other users belong.

(A2) Each user behaves strategically, that is, each user is not a price-taker. The users's objective is to maximize his own utility function.
(A3) The network manager knows the topology and resources of the network. This knowledge is the manager's private information. The network manager is not a profit-maker (i.e. he does not have a utility function).

(A4) The network manager receives requests for service from the network users. Based on these requests, he announces to each user $\left(j, G_{i}\right)$,

1) The multicast group to which the user belongs.

2) The set of links that form user $\left(j, G_{i}\right)$ 's route, $\mathcal{R}_{\left(j, G_{i}\right)}$.

3) The capacity of each link in $\mathcal{R}_{\left(j, G_{i}\right)}$.

(A5) Based on the network manager's announcement, each strategic user competes for resources (bandwidth) at each link of his route with the other users in that link ${ }^{2}$.

From the above description it is clear that the information in the network is decentralized. Every user in each group only knows his own utility but does not know the other users' utilities or the network's topology and its resources. The network manager knows the network's topology and its resources, but does not know the users' utilities. It is also clear that the network manager (which is not profit maker) acts like an accountant who sets up the users' routes, specifies the users competing for resources/bandwidth at each link, collects the money from the users $\left(j, G_{i}\right)$ that pay tax (i.e. $\left.t_{\left(j, G_{i}\right)}>0\right)$ and distributes it to those users who receive money.

As a consequence of assumptions (A1)-(A5) we have at each link of the network a decentralized resource allocation problem which can be studied/analyzed within the context of implementation theory [18]. These decentralized resource allocation problems are not independent/decoupled, as the rate that each user receives at any link of his own route must be the same. This constraint is dictated by the nature of the multi-rate multicast service provisioning problem and has a direct implication on the nature of the mechanism/game form we present in section III.

Under the above assumptions the objective is to determine a game form/mechanism which has the following properties: for each realization $\left(T, U_{\left(j, G_{i}\right)}, j \in G_{i}, G_{i} \in \mathcal{N}\right)$,

(P1) All Nash equilibria (NE) of the game induced by the mechanism result in allocations that are optimal solutions of the corresponding centralized problem Max.1.

(P2) The mechanism is individually rational, that is, the network users voluntarily participate in the decentralized bandwidth allocation process.

(P3) The budget is balanced at every equilibrium point of the game induced by the game form as well as off equilibrium.

\footnotetext{
${ }^{2}$ Since in this paper we present decentralized resource allocation mechanisms in equilibrium form, it is reasonable to assume that during the play of the game at each link $l \in \mathbf{L}$, each user of link $l$ learns the set of the other users competing for bandwidth at $l$.
} 
Before we proceed with the specification of our game form/mechanism we comment on the appropriateness of $\mathrm{NE}$ as an equilibrium/solution concept for the decentralized resource allocation problem under consideration. Two aspects/features of the problem and the solution we seek are crucial in establishing NE as an appropriate solution concept. (F1) The fact that the problem's environment (i.e. the users' utilities, the network topology and resources) is stable, that is, it does not change before the network users/agents reach their equilibrium behavior. (F2) The fact that we are looking for mechanisms in equilibrium form that possesses (P1)-(P3). Because of these features NE is an appropriate solution concept for the problem under consideration. As Nash points out in his "mass-action" interpretation of NE points, (which is valid under (F1)-(F2)), "It is unnecessary to assume that participants have full knowledge of the total structure of the game...but the participants are supposed to accumulate empirical information on the relative advantages of the various pure strategies at their proposal", J. Nash, PhD thesis ( [19] pg. 21). An interpretation similar to that of Nash also appears in [21] (pp. 69-70) and in [20] where the authors state: "We interpret our analysis as applying to an unspecified (message exchange) process in which users grope their way to a stationary message and in which the Nash property is a necessary condition for stationarity". In this paper we adopt $\mathrm{NE}$ as an equilibrium/solution concept, and interpret it in the same way as in [19]-[21]. A philosophy similar to ours has also been adopted in [22]-[24].

\section{Key features/natures of the problem}

Multi-rate multicast service provisioning with strategic users is the combination of a market problem and a public goods problem. Thus, the model as well as the allocation problem are new, even within the context of the mechanism design. Specifically, resource allocation among groups is a market problem; resource allocation among the users of the same group is a public goods problem.

The market component: One can see that bandwidth allocation among groups is a market problem as follows. One can consider a group as a single agent. The demand of this group at each link of the network is the maximum of demands of the users of the group on that link. So, with each group considered a single agent/singleton the multi-rate multicast service provisioning problem with strategic users becomes equivalent to the unicast service provisioning problem with strategic users. It is well known [22] that the unicast service provisioning problem with strategic users is a market problem with strategic users. At each link, the price per unit of bandwidth paid collectively by each group ${ }^{3}$ using the link is the same.

The public goods component: One can see that the resource allocation problem among the users of the same group is a public goods problem as follows. At equilibrium, the group receives at each link of the network a bandwidth/rate equal to the maximum requested by a user in the group. Each

\footnotetext{
${ }^{3}$ The price per unit of bandwidth paid collectively by each multicast group at a link $l$ is equal to the sum of the prices paid by the members' of the group who use the link $l$.
}

user of the group receives, in general, different rate, and the members of the group that use the link must collectively pay the price per unit of bandwidth charged at the link. At each link, each user of a group using the link contributes, in general, a different percentage of the price per of unit of bandwidth charged at that link; this percentage depends on the amount of bandwidth received by the user, the user's utility, and the number of users that are present in the group and use the link. Consequently, the resource allocation problem along users of the same group is a public goods problem.

In the following two sections we present a mechanism/game form for the problem formulated in this section and prove that it possess properties (P1-P3) stated in section II-B.

\section{A Mechanism For Rate Allocation}

Based on the characteristics of the multi-rate multicast problem, we present guidelines for the design of rate allocation mechanisms in section III-A. In section III-B, we specify a mechanism/game form for the decentralized rate allocation problem formulated in section II. In section III-C, we discuss and interpret the components of the mechanism.

\section{A. Guidelines for the design of the mechanism}

In section II-C we pointed out that the multi-rate multicast problem with strategic users is the combination of a market problem and a public goods problem. Therefore, the mechanism for rate allocation must capture both aspects/components of the problem. We now discuss the attributes a mechansim must have so that it can capture the market component and the public goods component of the multi-rate multicast problem.

To address the market characteristics of the problem the mechanism must be such that:

(i) All groups that use a particular link must pay the same price per unit of bandwidth at the link.

(ii) The bandwidth allocation to groups at each link must satisfy the link's capacity constraint.

(iii) The budget must be balanced, that is the sum of payments of all the groups that use the network must be equal to zero at equilibrium and off equilibrium.

To address the public goods characteristics of the problem the mechanism must be such that:

(iv) At any link $l$, different users of the same group that use the link pay, in general, different prices per unit of bandwidth at link $l$. Specifically: if user $a$ of group $G$ requires more bandwidth than user $b$ of group $G$ at link $l$, user $a$ must not pay less per unit of bandwidth at link $l$ than user $b$. In general, if users $a$ and $b$ require the same amount of bandwidth at link $l$, they do not necessarily pay the same price per unit of bandwidth at $l$ because they may have different utility functions.

(v) The price that user $i$ of group $G$ pays per unit of bandwidth at a particular link that he uses must not be under his control; that is, the price must be determined by the messages/strategies of the other users that use the same 
link. This feature of the mechanism is a consequence of the users' strategic behavior.

With these considerations in mind we proceed to specify our mechanism.

\section{B. Specification of the mechanism}

A game form/mechanism ( [18], [22], [23]) consists of two components $\mathcal{M}, f$. The component $\mathcal{M}$ denotes the users' message/strategy space, $\mathcal{M}$ defines the information the users are allowed to communicate with one another during the message exchange process. The component $f$ is the outcome function; $f$ defines for every message/strategy profile, the bandwidth/rate allocated to each user and the tax (subsidy) each user pays (receives). For the decentralized resource allocation problem formulated in section II we propose a game form/mechanism the components of which we describe below.

Message space: The message/strategy space for user $\left(j, G_{i}\right)$, $j \in G_{i}, G_{i} \in \mathcal{N}$, is given by $\mathcal{M}_{\left(j, G_{i}\right)}=\mathbb{R}_{+}^{\left|\mathcal{R}_{\left(j, G_{i}\right)}\right|+1}$. Specifically, a message of user $j$ is of the form

$$
m_{\left(j, G_{i}\right)}=\left[x_{\left(j, G_{i}\right)}, \pi_{\left(j, G_{i}\right)}^{l_{j_{1}}}, \pi_{\left(j, G_{i}\right)}^{l_{j_{2}}}, \cdots, \pi_{\left(j, G_{i}\right)}^{l_{j} \mathcal{R}_{\left(j, G_{i}\right)} \mid}\right],
$$

where $\left|\mathcal{R}_{\left(j, G_{i}\right)}\right|$ denotes the number of links along the route $\mathcal{R}_{\left(j, G_{i}\right)}$. The component $x_{\left(j, G_{i}\right)}$ denotes the bandwidth/rate user $\left(j, G_{i}\right)$ requests at all the links of his route. The component $\pi_{\left(j, G_{i}\right)}^{l_{j_{k}}} \in[0, \Upsilon]^{4}, 0 \leq \Upsilon<\infty, k=1,2, \cdots,\left|\mathcal{R}_{\left(j, G_{i}\right)}\right|$, denotes the price per unit of bandwidth user $\left(j, G_{i}\right)$ is willing to pay at link $l_{j_{k}}$ of his route.

Remark 1: Due to the nature of the multi-rate multicast service provisioning problem (see section II) the bandwidth/rate allocated to any user $\left(j, G_{i}\right), j \in G_{i}, G_{i} \in \mathcal{N}$, must be the same at all links of his route. Thus, the nature of message $m_{\left(j, G_{i}\right)}$ is a consequence of the above requirement.

Outcome Function: The outcome function $f$

$$
\begin{aligned}
f: \times_{G_{i} \in \mathcal{N}} \times_{j \in G_{i}} \mathcal{M}_{\left(j, G_{i}\right)} \rightarrow & \\
& \mathbb{R}_{+}^{\sum_{G_{i} \in \mathcal{N}}\left|G_{i}\right|} \times \mathbb{R}^{\sum_{G_{i} \in \mathcal{N}} \sum_{\left(j, G_{i}\right) \in G_{i}}\left|\mathcal{R}_{\left(j, G_{i}\right)}\right|}
\end{aligned}
$$

is defined as follows: for any

$$
m:=\left(m_{i \in G_{1}}, m_{j \in G_{2}}, \cdots, m_{k \in G_{N}}\right) \in \mathcal{M}
$$

where

$$
\begin{aligned}
& \mathcal{M}:=\times_{G_{i} \in \mathcal{N}} \times_{j \in G_{i}} \mathcal{M}_{\left(j, G_{i}\right)}, \\
f(m)= & f\left(m_{i \in G_{1}}, m_{j \in G_{2}}, \cdots, m_{k \in G_{N}}\right) \\
= & \left(\left(x_{\left(i, G_{1}\right)}, t_{\left(i, G_{1}\right)}\right), \cdots,\left(x_{\left(k, G_{N}\right)}, t_{\left(k, G_{N}\right)}\right)\right),
\end{aligned}
$$

where $t_{\left(j, G_{i}\right)}:=\left(t_{j}^{l_{j_{1}}}, t_{j}^{l_{j_{2}}}, \cdots, t_{j}^{l_{j_{\mathcal{R}_{j, G}} \mid}}\right)$, for every $\left(j, G_{i}\right)$, $j \in G_{i}, G_{i} \in \mathcal{N}$, is the tax (subsidy) that user $\left(j, G_{i}\right)$ pays (receives) to (from) the other users, through the network manager, for each link $l_{j_{k}} \in \mathcal{R}_{\left(j, G_{i}\right)}$, and $x_{\left(j, G_{i}\right)}, j \in G_{i}, G_{i} \in \mathcal{N}$, represents the amount of

\footnotetext{
${ }^{4}$ For technical resons (c.f. Theorem 5) we choose $\Upsilon$ to be arbitrary and large but finite.
}

bandwidth/rate allocated to user $\left(j, G_{i}\right)$.

The tax $t_{j}^{l_{j_{k}}}, k=1,2, \cdots,\left|\mathcal{R}_{\left(j, G_{i}\right)}\right|, \forall j \in G_{i}, G_{i} \in \mathcal{N}$, is defined in accordance with the number of multicast groups using link $l$. We consider four cases.

- Case A. $\left|Q_{l}\right|=1$

Let $Q_{l}=\left\{G_{\zeta}\right\}$. Then, for any $j \in G_{\zeta}(l)$,

$$
\begin{aligned}
t_{\left(j, G_{\zeta}\right)}^{l}= & \mathbb{I}\left\{x_{\left(j, G_{\zeta}\right)}=x_{G_{\zeta}}(l)\right\} \\
& \times\left\{0 \cdot \mathbb{I}\left\{x_{G_{\zeta}}(l) \leq c_{l}\right\}+\frac{1\left\{x_{G_{\zeta}}(l)>c_{l}\right\}}{1-1\left\{x_{G_{\zeta}}(l)>c_{l}\right\}}\right\} .
\end{aligned}
$$

The function $\mathbb{I}\{\cdot\}$ denotes the indicator function, i.e.,

$$
\mathbb{I}\{A\}= \begin{cases}1 & \text { if } A \text { holds } \\ 0 & \text { otherwise }\end{cases}
$$

The function $1\{A\}$, used throughout the paper, is defined as follows

$$
1\{A\}= \begin{cases}1-\epsilon & \text { if } A \text { holds } \\ 0 & \text { otherwise }\end{cases}
$$

where $\epsilon$ is bigger than zero and sufficiently small ${ }^{5} ; \epsilon$ is chosen by the mechanism designer.

- Case B. $\left|Q_{l}\right|=2$

Let $Q_{l}=\left\{G_{\zeta}, G_{\zeta+\mathbf{1}}\right\}$. We consider two subcases, $\left|G_{\zeta}^{\max }(l)\right| \geq 2$ and $\left|G_{\zeta}^{\max }(l)\right|=1$.

Part BI: $\left|G_{\zeta}^{\max }(l)\right| \geq 2$.

Let the label of $\left(j, G_{\zeta}\right)$ in $G_{\zeta}^{\max }(l)$ be $\left(k, G_{\zeta}^{\max }(l)\right)$. Then: If $\left(j, G_{\zeta}\right) \in G_{\zeta}^{\max }(l)$,

$$
\begin{aligned}
t_{\left(k, G_{\zeta}\right)}^{l}= & \pi_{\left(k+1, G_{\zeta}{ }^{\max }(l)\right)} x_{\left(j, G_{\zeta}\right)}+\frac{\Phi_{G_{\zeta} \max (l)}^{2}}{\alpha\left|G_{\zeta}{ }^{\max }(l)\right|} \\
& -2 \frac{P_{G_{\zeta+1}(l)^{\max }}}{\left|G_{\zeta}{ }^{\max }(l)\right|} \Phi_{G_{\zeta}{ }^{\max }(l)}\left[\frac{x_{G_{\zeta+1}}(l)+x_{\left(j, G_{\zeta}\right)}-c_{l}}{\gamma}\right] \\
& +\Xi_{\left(j, G_{\zeta}\right)}^{l}
\end{aligned}
$$

If $\left(k, G_{\zeta}\right) \notin G_{\zeta}^{\max }(l)$ then $t_{\left(k, G_{\zeta}\right)}^{l}=0$,

where $\alpha$ and $\gamma$ are sufficiently large constants, $P_{G_{\zeta}^{\max }(l)}=$ $\sum_{j \in G_{\zeta} \max (l)} \pi_{\left(j, G_{\zeta} \max (l)\right)}$, and $k+1$ is defined $\bmod \left(\left|G_{\zeta}^{\max }\right|\right)$, and

$$
\begin{aligned}
\Phi_{G_{\zeta}^{\max }(l)} & =P_{G_{\zeta} \max (l)}-P_{G_{\zeta+1} \max (l)} \\
\Xi_{\left(j, G_{\zeta}\right)}^{l} & =\frac{1\left\{x_{\left(j, G_{\zeta}\right)}>0\right\} 1\left\{x_{G_{\zeta+1}}(l)+x_{\left(j, G_{\zeta}\right)}-c_{l}>0\right\}}{1-1\left\{x_{\left(j, G_{\zeta}\right)}>0\right\} 1\left\{x_{G_{\zeta+1}}(l)+x_{\left(j, G_{\zeta}\right)}-c_{l}>0\right\}}
\end{aligned}
$$

Part BII: If $\left|G_{\zeta}{ }^{\max }(l)\right|=1$. Then: If $\left(j, G_{\zeta}\right) \in G_{\zeta}^{\max }(l)$,

$$
\begin{aligned}
t_{\left(j, G_{\zeta}\right)}^{l}= & P_{G_{\zeta+1}^{\max }(l)} x_{\left(j, G_{\zeta}\right)}+\frac{\Phi_{G_{\zeta} \max (l)}^{2}}{\alpha} \\
& -2 P_{G_{\zeta+1} \max }(l) \Phi_{G_{\zeta}{ }^{\max }(l)}\left[\frac{x_{G_{\zeta+1}}(l)+x_{\left(j, G_{\zeta}\right)}-c_{l}}{\gamma}\right] \\
& +\Xi_{\left(j, G_{\zeta}\right)}^{l}
\end{aligned}
$$

${ }^{5}$ Therefore, when $\mathrm{A}$ and $\mathrm{B}$ (both) hold, then $\frac{1\{A\} 1\{B\}}{1-1\{A\} 1\{B\}} \approx \frac{1}{0^{+}}$is well defined and it becomes a large number. 
If $\left(j, G_{\zeta}\right) \notin G_{\zeta}^{\max }(l)$ then $t_{\left(j, G_{\zeta}\right)}^{l}=0$.

- Case C. $\left|Q_{l}\right|=3$

Let $Q_{l}=\left\{G_{\zeta}, G_{\zeta+\mathbf{1}}, G_{\zeta+\mathbf{2}}\right\}$. We consider two subcases, $\left|G_{\zeta}^{\max }(l)\right| \geq 2$ and $\left|G_{\zeta}^{\max }(l)\right|=1$.

Part CI: $\left|G_{\zeta}{ }^{\max }(l)\right| \geq 2$. Then:

Let the label of $\left(j, G_{\zeta}\right)$ in $G_{\zeta}^{\max }(l)$ be $\left(k, G_{\zeta}^{\max }(l)\right)$. Then: If $\left(j, G_{\zeta}\right) \in G_{\zeta}^{\max }(l)$,

$$
\begin{aligned}
t_{\left(j, G_{\zeta}\right)}^{l}= & \pi_{\left(k+1, G_{\zeta} \max (l)\right)} x_{\left(j, G_{\zeta}\right)}+\frac{\bar{\Phi}_{G_{\zeta} \max (l)}^{2}}{\alpha\left|G_{\zeta}^{\max }(l)\right|} \\
& -2 \frac{P_{-G_{\zeta} \max (l)}}{\left|G_{\zeta}{ }^{\max }(l)\right|} \bar{\Phi}_{G_{\zeta} \max (l)}\left[\frac{\mathcal{E}_{-G_{\zeta} \max (l)}+x_{\left(j, G_{\zeta}\right)}}{\gamma}\right] \\
& +\bar{\Xi}_{\left(j, G_{i}\right)}^{l}
\end{aligned}
$$

If $\left(j, G_{\zeta}\right) \notin G_{\zeta}^{\max }(l)$ then $t_{\left(j, G_{\zeta}\right)}^{l}=0$.

Part CII: $\left|G_{\zeta}^{\max }(l)\right|=1$.

If $\left(j, G_{\zeta}\right) \in G_{\zeta}^{\max }(l)$,

$$
\begin{aligned}
t_{\left(j, G_{\zeta}\right)}^{l}= & P_{-G_{\zeta} \max (l)} x_{\left(j, G_{\zeta}\right)} \\
& -2 P_{-G_{\zeta} \max } \bar{\Phi}_{G_{\zeta}^{\max }(l)}\left[\frac{\mathcal{E}_{-G_{\zeta} \max (l)}+x_{\left(j, G_{\zeta}\right)}}{\gamma}\right] \\
& +\frac{\bar{\Phi}_{G_{\zeta} \max (l)}^{2}}{\alpha}+\bar{\Xi}_{\left(j, G_{i}\right)}^{l}
\end{aligned}
$$

If $\left(j, G_{\zeta}\right) \notin G_{\zeta}^{\max }(l)$ then $t_{\left(j, G_{\zeta}\right)}^{l}=0$, where

$$
\begin{aligned}
\mathcal{E}_{-G_{\zeta} \max (l)} & :=x_{G_{\zeta+1}}(l)+x_{G_{\zeta+2}}(l)-c_{l}, \\
P_{G_{\zeta}}{ }^{\max }(l) & :=\sum_{j \in G_{\zeta} \max ^{\max }(l)} \pi_{\left(j, G_{\zeta} \max (l)\right)}, \\
P_{-G_{\zeta} \max (l)} & :=\frac{P_{G_{\zeta+1}{ }^{\max }(l)}+P_{G_{\zeta+2}}{ }^{\max (l)}}{2}, \\
\bar{\Phi}_{G_{\zeta} \max (l)} & :=P_{G_{\zeta} \max (l)}-P_{G_{\zeta+1} \max (l)}, \\
\bar{\Xi}_{\left(j, G_{i}\right)}^{l} & :=\frac{1\left\{x_{\left(j, G_{\zeta}\right)}>0\right\} 1\left\{\mathcal{E}_{-G_{\zeta}^{\max }(l)}+x_{\left(j, G_{\zeta}\right)}>0\right\}}{1-1\left\{x_{\left(j, G_{\zeta}\right)}>0\right\} 1\left\{\mathcal{E}_{-G_{\zeta}^{\max }(l)}+x_{\left(j, G_{\zeta}\right)}>0\right\}}
\end{aligned}
$$

- Case D. $\left|Q_{l}\right|>3$

Let $G_{i} \in Q_{l}$. We consider two subcases, $\left|G_{i}{ }^{\max }(l)\right| \geq 2$ and $\left|G_{i}{ }^{\max }(l)\right|=1$.

Part DI: $\left|G_{i}{ }^{\max }(l)\right| \geq 2$.

Let the label of $\left(j, G_{i}\right)$ in $G_{i}^{\max }(l)$ be $\left(k, G_{i}^{\max }(l)\right)$. Then: If $\left(j, G_{i}\right) \in G_{i}^{\max }(l)$,

$$
\begin{aligned}
t_{\left(j, G_{i}\right)}^{l}= & \pi_{\left(k+1, G_{i} \max (l)\right)} x_{\left(j, G_{i}\right)}+\frac{\bar{\Phi}_{G_{i}^{\max }(l)}^{2}}{\left|G_{i}^{\max }(l)\right|} \\
& -2 \frac{P_{-G_{i} \max }}{\left|G_{i}{ }^{\max }(l)\right|} \bar{\Phi}_{G_{i}^{\max }(l)}\left[\frac{\mathcal{E}_{-G_{i} \max (l)}+x_{\left(j, G_{i}\right)}}{\gamma}\right] \\
& +\Xi_{\left(j, G_{i}\right)}+\frac{\Gamma_{G_{i}}^{l}}{\left|G_{i}^{\max }(l)\right|}
\end{aligned}
$$

If $\left(j, G_{i}\right) \notin G_{i}^{\max }(l)$ then

$$
t_{\left(j, G_{i}\right)}^{l}=0 .
$$

where $\mathcal{E}_{-G_{i}{ }^{\max }(l)}, P_{G_{i} \max (l)}$, and $P_{-G_{i} \max (l)}$ are defined by equations similar to (18)-(20).

Part DII: $\left|G_{i}{ }^{\max }(l)\right|=1$.

If $\left(j, G_{i}\right) \in G_{i}^{\max }(l)$,

$$
\begin{aligned}
t_{\left(j, G_{i}\right)}^{l}= & P_{-G_{i} \max } x_{j, G_{i}}+\bar{\Phi}_{G_{i}^{\max }(l)}^{2} \\
& -2 P_{-G_{i} \max } \bar{\Phi}_{G_{i}^{\max }(l)}\left[\frac{\mathcal{E}_{-G_{i} \max (l)}+x_{\left(j, G_{i}\right)}}{\gamma}\right] \\
& +\Xi_{\left(j, G_{i}\right)}+\Gamma_{G_{i}}^{l}
\end{aligned}
$$

If $\left(j, G_{i}\right) \notin G_{i}^{\max }(l)$ then

$$
t_{\left(j, G_{i}\right)}^{l}=0,
$$

where,

$$
\begin{aligned}
& \mathcal{E}_{-G_{i} \max (l)}:=\left[\sum_{\substack{G_{k} \in Q_{l} \\
G_{k} \neq G_{i}}} x_{G_{k}}(l)\right]-c_{l}, \\
& P_{G_{i} \max (l)}:=\sum_{j \in G_{i} \max (l)} \pi_{\left(j, G_{i} \max (l)\right)}, \\
& P_{-G_{i} \max (l)}:=\frac{\sum_{\substack{G_{k} \in Q_{l} \\
G_{k} \neq G_{i}}} P_{G_{k} \max (l)}}{\left|Q_{l}\right|-1} \\
& =\frac{\sum_{\substack{G_{k} \in Q_{l} \\
G_{k} \neq G_{i}}} \sum_{j \in G_{k} \max (l)} \pi_{\left(j, G_{k} \max (l)\right)}}{\left|Q_{l}\right|-1},
\end{aligned}
$$

$\Gamma_{G_{i}}^{l}:=$

$$
\begin{aligned}
& \frac{\sum \sum_{\substack{G_{s}, G_{r} \in Q_{l} \\
G_{r} \neq G_{s} \neq G_{i}}}\left(2 P_{G_{s}} \max (l) P_{G_{r} \max (l)}\left(1+\frac{x_{G_{s}}(l)}{\gamma}\right)\right)}{\left(\left|Q_{l}\right|-1\right)\left(\left|Q_{l}\right|-2\right)} \\
& 2 \sum \sum \sum \sum_{\substack{G_{s}, G_{r}, G_{t} \in Q_{l} \\
G_{t} \neq G_{s} \neq G_{r} \neq G_{i}}} P_{G_{r} \max (l)} P_{G_{s}} \max ^{\max (l)} \mathcal{E}_{G_{t} \max (l)} \\
& +\frac{G_{t} \neq G_{s} \neq G_{r} \neq G_{i}}{\left(\left|Q_{l}\right|-1\right)^{2}\left(\left|Q_{l}\right|-3\right) \gamma} \\
& 2 \sum \sum \sum \sum_{\substack{G_{s}, G_{r}, G_{t} \in Q_{l} \\
G_{t} \neq G_{s} \neq G_{r} \neq G_{i}}} P_{G_{r} \max (l)} P_{G_{t} \max (l)} x_{G_{s}}(l) \\
& \left(\left|Q_{l}\right|-1\right)^{2}\left(\left|Q_{l}\right|-3\right) \gamma \\
& 2 \sum \sum_{G_{s}, G_{r} \in Q_{l}} P_{G_{r} \max (l)} P_{G_{s}{ }^{\max }(l)} \mathcal{E}_{G_{r} \max (l)} \\
& +\frac{G_{s} \neq G_{r} \neq G_{i}}{\left(\left|Q_{l}\right|-1\right)^{2}\left(\left|Q_{l}\right|-2\right) \gamma} \\
& \frac{2 \sum \sum_{\substack{G_{s}, G_{r} \in Q_{l} \\
G_{s} \neq G_{r} \neq G_{i}}} P_{G_{r} \max (l)} P_{G_{r} \max (l)} x_{G_{s}}(l)}{\left(\left|Q_{l}\right|-1\right)^{2}\left(\left|Q_{l}\right|-2\right) \gamma} \\
& \left(\left|Q_{l}\right|-1\right)^{2}\left(\left|Q_{l}\right|-2\right) \gamma \\
& -\frac{2 P_{-G_{i} \max (l)}^{2} \mathcal{E}_{-G_{i} \max (l)}}{\gamma}-\frac{\sum_{G_{s} \in Q_{l}} P_{G_{s} \max (l)}{ }^{2}}{\left(\left|Q_{l}\right|-1\right)} \\
& -P_{-G_{i} \max (l)}^{2} .
\end{aligned}
$$

Next we specify additional subsidies $\mathcal{S}^{l}$ that user $\left(j, G_{i}\right), j \in G_{i}, G_{i} \in \mathcal{N}$, may receive. For that matter we consider all links $l \in \mathbf{L}$ where $\left|Q_{l}\right| \leq 3$. For each such link $l$, we define the quantity

$$
\begin{aligned}
\mathcal{S}^{l}:= & \sum_{G_{\zeta} \in Q_{l}} \sum_{\left(j, G_{\zeta}\right) \in G_{\zeta} \max (l)}-t_{\left(j, G_{\zeta}\right)}^{l} \mathbb{I}\{\text { Case } \quad \mathrm{B}\} \\
& +\sum_{G_{\zeta} \in Q_{l}} \sum_{\left(j, G_{\zeta}\right) \in G_{\zeta}{ }^{\max }(l)}-t_{\left(j, G_{\zeta}\right)}^{l} \mathbb{I}\{\text { Case } \quad \mathrm{C}\}(22)
\end{aligned}
$$


Since $\alpha$ and $\gamma$ are sufficiently large,

$$
\begin{aligned}
\mathcal{S}^{l}= & \left.o(1)-\sum_{G_{\zeta}, G_{\zeta} \in Q_{l}} P_{G_{\zeta}^{\max }(l)} x_{G_{\zeta}}(l) \mathbb{I}\{\text { Case B(Part BI })\right\} \\
& \left.-\sum_{G_{\zeta}, G_{\zeta} \in Q_{l}} P_{G_{\zeta}^{\max }(l)} x_{G_{\zeta}}(l) \mathbb{I}\{\text { Case C(Part CI })\right\} \\
& \left.-\sum_{G_{\zeta}, G_{\zeta} \in Q_{l}} P_{-G_{\zeta}^{\max }(l)} x_{G_{\zeta}}(l) \mathbb{I}\{\text { Case B(Part BII })\right\} \\
& \left.-\sum_{G_{\zeta}, G_{\zeta} \in Q_{l}} P_{-G_{\zeta}^{\max }(l)} x_{G_{\zeta}}(l) \mathbb{I}\{\text { Case C(Part CII })\right\} \\
:= & o(1)-S_{+}^{l} .
\end{aligned}
$$

For each $l \in \mathbf{L}$ where $\left|Q_{l}\right| \leq 3$ the network manager chooses at random a user $k_{l} \notin \bigcup_{G_{i} \in Q_{l}} G_{i}$ and assigns the subsidy $\mathcal{S}^{l}$ to user $k_{l}$. Let $l_{1}, l_{2}, \cdots, l_{r}$ be the set of links such that $\left|Q_{l_{i}}\right| \leq 3$, and let $k_{l_{i}}$ be the corresponding users that receive $\mathcal{S}^{l_{i}}$.

Based on the above, the tax (subsidy) paid (received) by user $\left(j, G_{i}\right), j \in G_{i}, G_{i} \in \mathcal{N}$, is the following. If $\left(j, G_{i}\right) \neq$ $k_{l_{1}}, k_{l_{2}}, \cdots k_{l_{r}}$ then

$$
t_{\left(j, G_{i}\right)}=\sum_{l \in \mathcal{R}_{\left(j, G_{i}\right)}} t_{\left(j, G_{i}\right)}^{l},
$$

where for each $l \in \mathcal{R}_{\left(j, G_{i}\right)}, t_{\left(j, G_{i}\right)}^{l}$ is determined in accordance with $\left|Q_{l}\right|$. If $\left(j, G_{i}\right)=k_{l_{n}}$ for some $k_{l_{n}} \in \bigcup_{m=1}^{r} k_{l_{m}}$, then

$$
t_{k_{l_{i}}}=\sum_{l \in \mathcal{R}_{k_{l_{i}}}} t_{k_{l_{i}}}^{l}+\mathcal{S}^{l_{i}},
$$

where $\mathcal{S}^{l_{i}}$ is defined by (22) and $\mathcal{R}_{k_{l_{i}}}$ is the set of links used by user $k_{l_{i}}$.

Note that $\mathcal{S}^{l_{i}}$ is not controlled by user $k_{l_{i}}$. Thus, the presence (or absence) of $\mathcal{S}^{l_{i}}$ does not influence the strategic behavior of user $k_{l_{i}}$. We have assumed here that the users $k_{l_{1}}, k_{l_{2}}, \cdots, k_{l_{r}}$, are distinct. Expressions similar to the above hold when the users $k_{l_{1}}, k_{l_{2}}, \cdots, k_{l_{r}}$ are not distinct.

\section{Discussion/Interpretation of the Mechanism}

We now interpret the mechanism presented in section III-B, based on the guidelines for its design, presented in section III-A. We focus on Case D (Part DI). The mechanism's interpretation is similar in all other cases. To proceed with the interpretation we define:

$$
\begin{aligned}
\Delta_{1}^{\left(j, G_{i}\right)}(l):= & \pi_{\left(k+1, G_{i}{ }^{\max }(l)\right)} x_{\left(j, G_{i}\right)}, \\
\Delta_{2}^{\left(j, G_{i}\right)}(l):= & \frac{\bar{\Phi}_{G_{i}^{\max }(l)}^{2}}{\left|G_{i}{ }^{\max }(l)\right|} \\
& -2 \frac{P_{-G_{i} \max }(l)}{\left|G_{i}{ }^{\max }(l)\right|} \bar{\Phi}_{G_{i}^{\max }(l)}^{2}\left[\frac{\mathcal{E}_{-G_{i} \max (l)}+x_{\left(j, G_{i}\right)}}{\gamma}\right] \\
& +\Xi_{\left(j, G_{i}\right)} \\
\Delta_{3}^{\left(j, G_{i}\right)}(l):= & \frac{\Gamma_{G_{i}}^{l}}{\left|G_{i}^{\max }(l)\right|} \\
\Delta_{4}^{\left(j, G_{i}\right)}(l):= & \mathbb{I}\left\{x_{\left(j, G_{i}\right)}=x_{G_{i}}(l)\right\} .
\end{aligned}
$$

Note that Eqs. (14) and (15) can be collectively rewritten as follows,

$t_{\left(j, G_{i}\right)}^{l}=\left[\Delta_{1}^{\left(j, G_{i}\right)}(l)+\Delta_{2}^{\left(j, G_{i}\right)}(l)+\Delta_{3}^{\left(j, G_{i}\right)}(l)\right] \times \Delta_{4}^{\left(j, G_{i}\right)}(l)$.

$\Delta_{1}^{\left(j, G_{i}\right)}(l), \Delta_{2}^{\left(j, G_{i}\right)}(l), \Delta_{3}^{\left(j, G_{i}\right)}(l)$, and $\Delta_{4}^{\left(j, G_{i}\right)}(l)$ collectively represent the tax (subsidy) user $\left(j, G_{i}\right)$ pays (receives) for using link $l$. The terms $\Delta_{1}^{\left(j, G_{i}\right)}(l)$ and $\Delta_{4}^{\left(j, G_{i}\right)}(l)$ (respectively, $\Delta_{2}^{\left(j, G_{i}\right)}(l)$ and $\left.\Delta_{3}^{\left(j, G_{i}\right)}(l)\right)$ capture/describe the public good (respectively, market) component of the problem.

We begin with the interpretation of the public good terms. Note that user $\left(j, G_{i}\right)$ pays taxes (receives subsidies) at link $l$ only if his bandwidth demand is the maximum among the users of group $G_{i}$ at link $l$. This is expressed by the term $\Delta_{4}^{\left(j, G_{i}\right)}(l)$. By assumption the cardinality of the set users from $G_{i}$ who have maximum bandwidth demand at link $l$ is greater than one. Assume now that $\left(j, G_{i}\right)$ is one of the users of group $G_{i}$ that have maximum bandwidth demand at link $l$, and let $\left(k, G_{i}^{\max }(l)\right)$ be the index of this user in $G_{i}^{\max }(l)$. The price per unit of bandwidth at link $l$ that this user pays is not under his control; it is determined by the message/strategy $\left(\pi_{\left(k+1, G_{i}\right.} \max _{(l))}\right)$ of user $\left(k+1, G_{i}^{\max }(l)\right)$, that is user $k+1$ of the group $G_{i}^{\max }(l)^{6}$. This is reflected in the term $\Delta_{1}^{\left(j, G_{i}\right)}(l)$ which represents the amount of tax user $\left(j, G_{i}\right)$ pays for the bandwidth he receives at link $l$. The two terms are consistent with the design guidelines associated with the public good features of the mechanism presented in section III-A. Specifically, terms $\Delta_{1}^{\left(j, G_{i}\right)}(l)$ and $\Delta_{4}^{\left(j, G_{i}\right)}(l)$ demonstrate that: (i) at any link $l$, if user $a$ of group $G_{i}$ receives more bandwidth than user $b$ of the same group, then user $a$ pays no less for this bandwidth than user $b$; (ii) if two users $a$ and $b$ of the same group require maximum amount of bandwidth at link $l$ they do not necessarily pay the same price per unit of bandwidth at that link. As a result of the specification and interpretation of the terms $\Delta_{1}^{\left(j, G_{i}\right)}(l)$ and $\Delta_{4}^{\left(j, G_{i}\right)}(l)$, the price group $G_{i}$ pays per unit of bandwidth at link $l$ is the sum of the prices its users with maximum demand at link $l$ pay. That is,

$$
P_{G_{i}^{\max }(l)}=\sum_{\left(j, G_{i}^{\max }(l)\right) \in G_{i}^{\max }(l)} \pi_{\left(j, G_{i}^{\max }(l)\right)} .
$$

We continue with interpretation of the market terms of the tax function. The term $\Delta_{2}^{\left(j, G_{i}\right)}(l)$ provides the following incentives to the groups using link $l$ : (1) To bid/propose the same price per unit of bandwidth at that link. (2) To collectively request a total bandwidth that does not exceed the capacity of the link. The incentive provided to all groups to bid the same price per unit of bandwidth is described by the term $\frac{\bar{\Phi}_{G_{i}^{\max }(l)}}{\left|G_{i} \max (l)\right|}=\frac{\left(P_{G_{i}} \max -P_{-G_{i}} \max \right)^{2}}{\left|G_{i} \max (l)\right|}$. The incentive provided to all users to collectively request a total bandwidth that does

\footnotetext{
${ }^{6}$ The situation where $\left(j, G_{i}\right)$ is the only user of group $G_{i}$ with the maximum demand at link $l$ is discussed in other cases (e.g. Case D (Part DII)), where it is shown again that the price user $\left(j, G_{i}\right)$ pays per unit of bandwidth at link $l$ is not controlled by him.
} 
not exceed the link's capacity is captured by the term

$$
\Xi_{\left(j, G_{i}\right)}=\frac{1\left\{x_{\left(j, G_{i}\right)}>0\right\} 1\left\{\mathcal{E}_{-G_{i}^{\max }(l)}+x_{\left(j, G_{i}\right)}>0\right\}}{1-1\left\{x_{\left(j, G_{i}\right)}>0\right\} 1\left\{\mathcal{E}_{-G_{i}^{\max }(l)}+x_{\left(j, G_{i}\right)}>0\right\}} .
$$

Note that each group is very heavily penalized if it requests a nonzero bandwidth at $l$, and, collectively, all the groups using $l$ request a total bandwidth that exceeds the link's capacity $c_{l}$. A joint incentive provided to all users to bid the same price per unit of bandwidth and to utilize the total capacity of the link is captured by the term

$$
-2 \frac{P_{-G_{i} \max }(l)}{\left|G_{i}{ }^{\max }(l)\right|} \bar{\Phi}_{G_{i}^{\max }(l)}\left[\frac{\mathcal{E}_{-G_{i} \max (l)}+x_{\left(j, G_{i}\right)}}{\gamma}\right] .
$$

The goal of the term $\Delta_{3}^{\left(j, G_{i}\right)}(l)$ is to lead to a balanced budget. It is important to note that the term $\Delta_{3}^{\left(j, G_{i}\right)}(l)$ is not controlled by group $G_{i}$, consequently, by any user in group $G_{i}$. Therefore, the presence of $\Delta_{3}^{\left(j, G_{i}\right)}(l)$ does not affect the behavior of any user of group $G_{i}$. The terms $\Delta_{2}^{\left(j, G_{i}\right)}(l)$ and $\Delta_{3}^{\left(j, G_{i}\right)}(l)$ are consistent with the guidelines that were presented in section III-A concerning the market features of the mechanism.

\section{Properties of the Mechanism}

We prove that the mechanism proposed in section III has the following properties. (P1) It implements the solution of problem Max.0 in Nash equilibria. (P2) It is individually rational. (P3) It is budget-balanced at every feasible allocation.

We establish the above properties by proceeding as follows. First, we prove that the game induced by the mechanism proposed in section III has at lest one pure NE (Theorem 1), and that all NE of the game induced by the game form/mechanism of section III result in feasible solutions of the centralized problem Max.0 (Theorem 2). Afterwards, we establish that the mechanism is budget-balanced at all feasible allocations. (Lemma 4). Then, we show that network users voluntarily participate in the allocation process. We do this by showing that each user's utility/payoff resulting from the allocations corresponding to all $\mathrm{NE}$ of the game induced by the mechanism is greater than or equal to zero, the payoff each user receives when he does not participate in the allocation process (Theorem 5). Finally, we show that the mechanism implements in Nash equilibria the solution of the centralized allocation problem Max.0 (Theorem 6).

We present the proofs of the following theorems and lemmas in the Appendix.

Theorem 1 (EXISTENCE OF NE): The game induced by the mechanism of section III has at lest one pure NE.

Theorem 2 (FEASIBILITY): If $m^{*}=\left(x^{*}, \pi^{*}\right)$ is a NE of the game induced by the game form of section III and the users' utility functions, then the allocation $x^{*}$ is a feasible solution of problem Max.0.

The following lemma presents some key properties of NE prices and rates.

Lemma 3: Let $m^{*}=\left(x^{*}, \pi^{*}\right)$ be a NE of the game induced by game form of section III. Then for every $l \in \boldsymbol{L}$ and $G_{i} \in Q_{l}$, we have,

$$
\begin{aligned}
& P_{-G_{i} \max (l)}^{*}=P_{G_{i} \max (l)}^{*}=: P_{G^{\max }(l)}^{*} \forall G_{i} \in Q_{l} \\
& P_{G^{\max }(l)}^{*}\left[\frac{\mathcal{E}_{-G_{i} \max (l)}^{*}+x_{G_{i}}^{*}(l)}{\gamma}\right]=0
\end{aligned}
$$

For every user $\left(j, G_{i}{ }^{\max }(l)\right)$ where $G_{i} \in Q_{l}$, we have,

$$
\left.\frac{\partial t_{\left(j, G_{i}^{\max }(l)\right)}^{l}}{\partial x_{G_{i}}(l)}\right|_{m=m^{*}}= \begin{cases}\pi_{\left(j+1, G_{i} \max (l)\right)}^{*}, & \text { if }\left|G_{i}^{\max }(l)\right| \geq 2, \\ P_{G^{\max }(l)}^{*}, & \text { otherwise, }\end{cases}
$$

and

$\left[\frac{\partial U_{\left(j, G_{i}^{\max }(l)\right)}\left(x_{\left(j, G_{i}^{\max }(l)\right)}\right)}{\partial x_{\left(j, G_{i}^{\max }(l)\right)}}-\sum_{l \in \mathcal{R}_{\left(j, G_{i}\right)}^{\max }} \frac{\partial t_{\left(j, G_{i}^{\max }(l)\right)}^{l}}{\partial x_{\left(j, G_{i}^{\max }(l)\right)}}\right]_{m=m^{*}}=0$.

An immediate consequence of Lemma 3 and the specification of the tax for each user, defined by Eqs. (10)-(25), is the following.

Corollary 1: At every $N E m^{*}$ of the mechanism the tax function has the following form,

$t_{\left(j, G_{i}^{\max }(l)\right)}^{l}\left(m^{*}\right)=$

$\begin{cases}\pi_{\left(j+1, G_{i}^{\max }(l)\right)}^{*} x_{G_{i}}^{*}(l) & \text { Case B, Part BI; } \\ P_{G^{\max }}^{*} x_{G_{i}}^{*}(l) & \text { Case B, Part BII; } \\ \pi_{\left(j+1, G_{i}^{\max }(l)\right)}^{*} x_{G_{i}}^{*}(l) & \text { Case C, Part CI; } \\ P_{G^{\max }}^{*} x_{G_{i}}^{*}(l) & \text { Case C, Part CII; } \\ \pi_{\left(j+1, G_{i}^{\max }(l)\right)}^{*} x_{G_{i}}^{*}(l)-\frac{P_{G^{\max }(l)}^{*} x_{-G_{i}}(l)^{*}}{\left|G_{i}^{\max }\right|} & \text { Case D, Part DI; } \\ P_{G^{\max (l)}}^{*}\left(x_{G_{i}}^{*}(l)-x_{-G_{i}}^{*}(l)\right) & \text { Case D, Part DII }\end{cases}$

where

$$
x_{-G_{i}}^{*}(l)=\frac{\sum_{\substack{G_{j} \\ G_{j} \neq G_{i} \\ G_{j} \in Q_{l}}} x_{G_{j}}^{*}(l)}{\left|Q_{l}\right|-1} .
$$

When $\left(j, G_{i}\right) \notin G_{i}^{\max }(l), t_{\left(j, G_{i}\right)}^{l}\left(m^{*}\right)=0$. Therefore,

$$
t_{\left(j, G_{i}\right)}\left(m^{*}\right)=\sum_{l \in \mathcal{R}_{\left(j, G_{i}\right)}^{\max }} t_{\left(j, G_{i}\right)}^{l}\left(m^{*}\right),
$$

for $\left(j, G_{i}\right) \neq k_{l_{1}}, k_{l_{2}}, \cdots, k_{l_{r}}$, (cf. section III), and for $j=k_{l_{s}}, s=1,2, \cdots, r$,

$$
t_{\left(j, G_{i}\right)}\left(m^{*}\right)=\sum_{l \in \mathcal{R}_{\left(j, G_{i}\right)}^{\max }} t_{\left(j, G_{i}\right)}^{l}\left(m^{*}\right)-S_{+}^{* j}
$$

In the following lemma, we prove that the proposed mechanism is always budget balanced.

Lemma 4: The proposed mechanism/game form is budget balanced at every feasible allocation. That is, the mechanism is budget-balanced at all allocations corresponding to NE messages as well as to all off-equilibrium messages/strategies that result in feasible allocations.

The next result asserts that the mechanism/game form proposed in section III is individually rational.

Theorem 5: (INDIVIDUAL RATIONALITY): The game form specified in section III is individually rational, i.e., at every $N E$ the corresponding allocation $\left(x^{*}, t^{*}\right)$ is weakly preferred 
by all users to zero, the payoff each user receives when he does not participate in the allocation process.

In the following theorem we show that every NE of the game induced by the game form proposed in section III is efficient.

Theorem 6: (NASH IMPLEMENTATION): The allocation $\left(f\left(m^{*}\right)=\left(x^{*}, t^{*}\right)\right)$ corresponding to a NE message $m^{*}$ is an optimal solution of the centralized problem Max.0.

\section{CONCLUSION}

We have proposed a mechanism for rate/bandwidth allocation in multi-rate multicast service provisioning and performed an equilibrium analysis of the mechanism. We discovered that when the users' utilities are concave, the mechanism possesses the following properties: (i) It implements in Nash equilibria the solution of the corresponding centralized rate allocation problem. (ii) It is individually rational. (iii) It is budgetbalanced at all feasible allocations.

We don't have an algorithm/tatonnement process for the computation of NE of the game induced by our mechanism. The discovery of such an algorithm is an important open problem in decentralized resource allocation problems with strategic users who possess private information. The main difficulty in addressing this open problem is the following. Consider an algorithm for our problem. At each stage of the algorithm each user updates his strategy/message. Since the users' utilities are not common knowledge, after each update a user, say user $i$, can report any strategy he deems to be beneficial for himself; that is, user $i$ can misreport/misrepresent his update and the other users may not be able to check whether or not user $i$ is following the rules of the algorithm. Consequently, the algorithm must provide incentives to the users/agents to follow its rules at each one of its stages. Such a provision of incentives must be based, in general, on all the information available at the current stage, and must take the whole future into account. We have not been able to discover an algorithm with the above feature.

To the best of our knowledge, multi-rate multicast service provisioning with strategic users provides the first instance that is a combination of a market and a public goods problem. Thus, the results of this paper are also a contribution to the state of the art of mechanism design.

\section{REFERENCES}

[1] S. Sarkar and L. Tassiulas, "Fair Allocation of Utilities in Multirate, Multicast Networks: A framework for unifying diverse fairness objectives," IEEE Transactions on Automatic Control, vol. 47, no. 6, pp. 93944, June 2002.

[2] S. Sarkar and L. Tassiulas, "A Framework for Routing and Congestion Control for Multicast Information Flows," IEEE Transactions on Information Theory, vol. 48, no. 10, pp. 2690-2708, October 2002.

[3] S. Sarkar and L. Tassiulas, "Layered multicast rate control based on Lagrangian relaxation and dynamic programming," IEEE Journal on Selected Areas in Communications, vol 24, no 8, pp. 1464-1474, 2006.

[4] K. Kar and S. Sarkar and L. Tassiulas, "A scalable low-overhead rate control for multi-rate multicast sessions," IEEE Journal on Selected Areas in Communications, vol 20, no 8, pp. 1541-1557, 2002.

[5] K. Kar and L. Tassiulas, 'Layered Multicast Rate Control based on Lagrangian Relaxation and Dynamic Programming," IEEE Journal on Selected Areas in Communications, vol. 24, no. 8, pp. 1464-1474, 2006.
[6] D. Rubenstein, J. Kurose, and D. Towsley, "The impact of multicast layering on network fairness," In Proc. of ACM-SIGCOMM, Cambridge, 1999.

[7] E. Graves, R. Srikant, and D. Towsley, "Decentralized computation of weighted max-min fair bandwidth allocation in networks with multicast flows," In: Proceedings Tyrrhenian international workshop on digital communications (IWDC), Taormina, Italy.

[8] J. Shapiro, D. Towsley, and J. Kurose, "Optimization-based congestion control for multicast communications, In Proc. of INFOCOM,Tel Aviv, Israel, 2000.

[9] H. Tzeng and K. Siu, "On max-min fair congestion for multicast ABR service in ATM, IEEE Journal of Selected areas of Communication, vol. 15, no. 3, pp. 545-556, 1997.

[10] D. Deb and R. Srikant, "Congestion control for fair resource allocation in networks with multicast flows, IEEE/ACM Transaction on Networking, vol. 12, no. 2, pp. 261-273, 2004.

[11] E. Zegura, "Routing algorithms in multicast switching topologies, In Proc. of Allerton Conference on Communication, Control and Computing, Monticello, 1993.

[12] W. Park, H. Owen, and E. Zegura, "Sonet/sdh multicast routing algorithms in symmetrical three-stage networks, In Proc. of International Communication Conference (ICC), Seattle, 1993.

[13] M. Donahoo and E. Zegura, "Center selection and migration for widearea multicast routing, Journal of High Speed Networks, vol. 6, no. 2, pp. 141-164, 1997.

[14] R. Gupta and J. Walrand, "Average bandwidth and delay for reliable multicast," In Proc. of IFIP Performance, Istanbul, Turkey, 1999.

[15] N. Duffield, J. Horowitz, D. Towsley, W. Wei, and T. Friedman, "Multicast-based loss inference with missing data," IEEE J Selected Areas in Communication, vol. 20, no. 4, pp. 700-713, 2002.

[16] T. Stoenescu, M. Liu and D. Teneketzis, "Multi-rate Multicast Service Provisioning, Part I: An Algorithm for Optimal Price Splitting Along Multicast Trees", Mathematical Methods of Operations Research, vol. 65, no. 2, pp. 199-228, 2007.

[17] T. Stoenescu, M. Liu and D. Teneketzis, "Multirate Multicast Service Provisioning, Part II: A Tatonnement Process for Rate Allocation," Mathematical Methods of Operations Research, vol. 65, no. 3, pp. 389415, 2007.

[18] L. Hurwicz and S. Reiter, Designing Economics Mechanisms. Cambridge University Press, 2006.

[19] J. F. Nash, Non-Cooperative Gams, PhD Thesis, Department of Mathmatics, Princeton University. May, 1950.

[20] S. Reichelstein and S. Reiter, "Game forms with minimal strategy spaces, Econometrica, vol. 56, no. 3, pp. 661-692, 1988.

[21] T. Groves and J. Ledyards, "Incentive compatibility since 1972, Information, Incentives, and Economics Mechanisms, Essays in Honor of Leonid Hurwics, pp. 48-111, 1987.

[22] A. Kakhbod and D. Teneketzis, "An efficient game form for unicast service provisioning," IEEE Transactions on Automatic Control, vol 57, no 2, pp. 392-404, 2012.

[23] A. Kakhbod and D. Teneketzis, "Power allocation and spectrum sharing in multi-user, multi-channel systems with strategic users," IEEE Transactions on Automatic Control, To appear.

[24] S. Sharma and D. Teneketzis, "A Game-Theoretic approach to decentralized optimal power allocation for cellular networks," Telecommunication Systems Journal, Special Issue Dedicated to GameComm '08, vol 47, pp. 65-80, 2011.

[25] S. Boyd and L. Vandenberghe, Convex Optimization. Cambridge University Press, 2004.

[26] D. Fudenberg and J. Tirole, Game Theory, MIT Press, 1991.

\section{APPENDIX}

Proof of Theorem 1: We prove in Theorem 2 that any $\mathrm{NE}$ of the game induced by the mechanism of section III (if such an equilibrium exists) results in a feasible allocation of Problem Max.0. Therefore, we restrict to the space

$$
\mathcal{M}=\times_{G_{i} \in \mathcal{N}} \times_{j \in G_{i}} \mathcal{M}_{\left(j, G_{i}\right)}
$$

of strategies that result in feasible allocations of problem Max.0. Then, the users' utilities

$$
V_{\left(j, G_{i}\right)}\left(x_{\left(j, G_{i}\right)}, t_{\left(j, G_{i}\right)}\right)=U_{\left(j, G_{i}\right)}\left(x_{\left(j, G_{i}\right)}\right)-t_{\left(j, G_{i}\right)}
$$


$\left(j, G_{i}\right) \in G_{i}, G_{i} \in \mathcal{N}$ (where $t_{\left(j, G_{i}\right)}$ is specified by the game form of section III) are concave in $m_{\left(j, G_{i}\right)}=\left(x_{\left(j, G_{i}\right)}, \pi_{\left(j, G_{i}\right)}\right)$ and continuous in $m=\left(m_{\left(j, G_{i}\right)},\left(j, G_{i}\right) \in G_{i}, G_{i} \in \mathcal{N}\right)$. Furthermore, the message spaces $\mathcal{M}_{\left(j, G_{i}\right)}$ are compact, convex and nonempty. Therefore, by Gliksberg's theorem, [26], there exists a pure NE of the game $\left(\mathcal{M}, f, V_{\left(j, G_{i}\right)},\left(j, G_{i}\right) \in\right.$ $\left.G_{i}, G_{i} \in \mathcal{N}\right)$ induced by the game form of section III.

Proof of Theorem 2: By the construction of the mechanism $x_{\left(j, G_{i}\right)}^{*} \geq 0$ for all $\left(j, G_{i}\right), G_{i} \in \mathcal{N}$. Suppose that $x^{*}$ is such that the capacity constraint is violated at some link $l$ and $x_{G_{i}}^{*}(l)>0$. Consider an agent $\left(k, G_{i}\right) \in G_{i}^{\max }(l)$ whose index in $G_{i}^{\max }(l)$ is $\left(j, G_{i}^{\max }(l)\right)$ and change his strategy to $x_{\left(k, G_{i}\right)}=0$. Then

$$
V_{\left(k, G_{i}\right)}\left(m_{\left(k, G_{i}\right)}, m_{-\left(k, G_{i}\right)}^{*}\right)>V_{\left(j, G_{i}\right)}\left(m_{\left(k, G_{i}\right)}^{*}, m_{-\left(k, G_{i}\right)}^{*}\right),
$$

and this is in contradiction with the fact that $m^{*}$ is a NE. Consequently, every NE results in a feasible allocation of problem Max.0.

Proof of Lemma 3: We prove this lemma for Case D, Part DI. In a way similar to the following we can prove the assertion of the lemma for all other cases.

CASE D (PART DI): Consider $G_{i} \in Q_{l}$, and $\left(j, G_{i}^{\max }(l)\right) \in$ $G_{i}^{\max }(l)$.

Since user $\left(j, G_{i}^{\max }(l)\right)$ does not control $\Gamma_{\left(j, G_{i}\right)}$, then

$$
\frac{\partial \Gamma_{\left(j, G_{i}^{\max }(l)\right)}}{\partial \pi_{\left(j, G_{i}^{\max }(l)\right)}}=\frac{\partial \Gamma_{\left(j, G_{i}^{\max }(l)\right)}}{\partial x_{G_{i}}(l)}=0
$$

Therefore, we must have

$$
\begin{aligned}
\left.\frac{\partial t_{\left(j, G_{i}^{\max }(l)\right)}^{l}}{\left.\partial \pi_{\left(j, G_{i} \max (l)\right.}\right)}\right|_{m=m^{*}} & -2 \frac{P_{-G_{i} \max (l)}^{*}}{\left|G_{i}{ }^{\max }(l)\right|}\left(\frac{\mathcal{E}_{-G_{i} \max (l)}^{*}+x_{G_{i}}^{*}(l)}{\gamma}\right) \\
& +\frac{2}{\left|G_{i}{ }^{\max }(l)\right|} \bar{\Phi}_{G_{i}^{\max }(l)}^{*} \\
= & 0 .
\end{aligned}
$$

Define $\Delta_{\left(j, G_{i} \max (l)\right)}$ as follows,

$$
\begin{aligned}
\Delta_{\left(j, G_{i} \max (l)\right):=} & -\frac{P_{-G_{i} \max (l)}^{*}}{\left|G_{i}{ }^{\max }(l)\right|}\left(\frac{\mathcal{E}_{-G_{i} \max (l)}^{*}+x_{G_{i}}^{*}(l)}{\gamma}\right) \\
& +\frac{\bar{\Phi}_{G_{i}^{\max }(l)}^{*}}{\left|G_{i}{ }^{\max }(l)\right|} .
\end{aligned}
$$

Summing over all the users in $G_{i}^{\max }(l)$ and using (32) we obtain

$$
\begin{aligned}
\sum_{\left(j, G_{i}^{\max }\right) \in G_{i} \max (l)} \Delta_{\left(j, G_{i} \max (l)\right)}=- & P_{-G_{i} \max (l)}^{*}\left(\frac{\mathcal{E}_{-G_{i} \max (l)}^{*}+x_{G_{i}}^{*}(l)}{\gamma}\right) \\
+ & \bar{\Phi}_{G_{i}^{\max }(l)}^{*}=0 .
\end{aligned}
$$

Moreover, summing over all $\left|Q_{l}\right|$ multicast groups and using (32)-(34) we get

$$
\begin{aligned}
& \sum_{G_{i} \in Q_{l}} \sum_{\left(j, G_{i}^{\max }(l)\right) \in G_{i} \max (l)} \frac{\partial t_{\left(j, G_{i}^{\max }(l)\right)}^{l}}{\left.\partial \pi_{\left(j, G_{i} \max (l)\right.}\right)}= \\
& \sum_{G_{i} \in Q_{l}} \sum_{\left(j, G_{i}^{\max }(l)\right) \in G_{i} \max (l)} \Delta_{\left(j, G_{i} \max (l)\right)}=0 .
\end{aligned}
$$

Furthermore we note that

$$
\sum_{G_{i} \in Q_{l}} P_{G_{i} \max (l)}=\sum_{G_{i} \in Q_{l}} P_{-G_{i}}{ }^{\max (l)} .
$$

Equations (34)-(36) along with Theorem 1 and the fact that $P_{-G_{i} \max (l)}^{*} \geq 0$ for every $G_{i}, G_{i} \in Q_{l}$, imply that

$$
P_{-G_{i} \max (l)}^{*}\left(\frac{\mathcal{E}_{-G_{i} \max (l)}^{*}+x_{G_{i}}^{*}(l)}{\gamma}\right)=0, \quad \forall G_{i} \in Q_{l} .
$$

From Eqs. (34) and (37) it follows that

$$
P_{-G_{i} \max (l)}^{*}=P_{G_{i} \max (l)}^{*}=: P_{G^{\max (l)}}^{*}, \quad \forall G_{i} \in Q_{l} .
$$

Consequently,

$$
P_{G^{\max }(l)}^{*}\left(\frac{\mathcal{E}_{-G_{i} \max (l)}^{*}+x_{G_{i}}^{*}(l)}{\gamma}\right)=0 .
$$

Eqs. (38) and (39) along with (14) give

$$
\begin{gathered}
\left.\frac{\partial t_{\left(j, G_{i}^{\max }(l)\right)}^{l}}{\partial x_{G_{i}}(l)}\right|_{m=m^{*}}=\pi_{\left(j+1, G_{i} \max (l)\right)}^{*}-2 \frac{P_{-G_{i} \max (l)}^{*}}{\gamma\left|G_{i}{ }^{\max }(l)\right|} \bar{\Phi}_{G_{i}^{\max }(l)}^{*} \\
=\pi_{\left(j+1, G_{i} \max (l)\right)}^{*} .
\end{gathered}
$$

Proof of Lemma 4: Equation (28) together with (28) and (29) imply that $\sum_{\left(j, G_{i}\right)} \bigcup_{G_{i} \in \mathcal{N}} G_{i} t_{(j, G)}^{*}=\sum_{l \in \mathbf{L}} \sum_{G_{i} \in Q_{l}} \sum_{j \in G_{i}} t_{\left(j, G_{i}\right)}^{* l}=0$. Thus, the mechanism is budget balanced at allocations corresponding to NE. Now, we prove that the proposed mechanism is also budget balanced off equilibrium.

For that matter, we first consider links $l \in \mathbf{L}$ where $\left|Q_{l}\right|>3$. Based on Eqs. (19) and (20) we obtain for every $G_{i} \in Q_{l}$, $\sum_{\left(j, G_{i}\right) \in G_{i}(l)} t_{\left(j, G_{i}\right)}^{l}=$

$$
\begin{aligned}
& P_{G_{i} \max (l)} x_{G_{i}}(l)+\bar{\Phi}_{G_{i}^{\max }(l)}^{2}+\Gamma_{G_{i}}^{l} \\
& -2 P_{-G_{i}{ }^{\max }(l)} \bar{\Phi}_{G_{i}^{\max }(l)}\left(\frac{\mathcal{E}_{-G_{i} \max (l)}+x_{G_{i}}(l)}{\gamma}\right) .
\end{aligned}
$$

Furthermore, by little algebra, we can show that for every $l \in \mathbf{L}$ where $\left|Q^{l}\right|>3$ the following equalities hold,

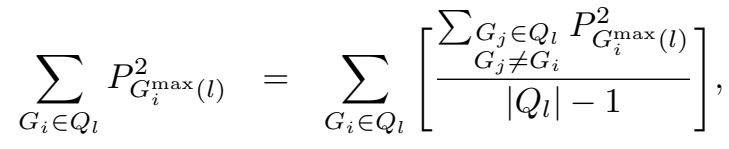

$$
\begin{aligned}
& \sum_{G_{i} \in Q_{l}}\left[2 P_{G_{i}^{\max }(l)} P_{-G_{i}^{\max }(l)}+2 P_{-G_{i}^{\max }(l)} P_{G_{i}^{\max }(l)} \frac{x_{G_{i}}(l)}{\gamma}\right] \\
& =\frac{\sum \sum \sum \sum_{G_{i}, G_{j}, G_{k} \in Q_{l}}\left(2 P_{G_{j}^{\max }(l)} P_{G_{k}^{\max }(l)}\left(1+\frac{x_{G_{j}}(l)}{\gamma}\right)\right)}{\left(\left|Q_{l}\right|-1\right)\left(\left|Q_{l}\right|-2\right)},
\end{aligned}
$$




$$
\begin{aligned}
& \sum_{G_{i} \in Q_{l}} P_{-G_{i}^{\max }(l)} P_{G_{i}^{\max }(l)} \frac{\mathcal{E}_{-G_{i}^{\max }(l)}}{\gamma}= \\
& \frac{\sum \sum \sum \sum_{\begin{array}{c}
G_{i}, G_{j}, G_{k}, G_{r} \in Q_{l} \\
G_{i} \neq G_{j} \neq G_{k} \neq G_{r}
\end{array}} 2 P_{G_{k}^{\max }(l)} P_{G_{j}^{\max }(l)} \mathcal{E}_{G_{r}^{\max }(l)}}{\gamma\left(\left|Q_{l}\right|-1\right)^{2}\left(\left|Q_{l}\right|-3\right)} \\
& \sum \sum \sum \sum_{G_{i}, G_{j}, G_{k} \in Q_{l}} 2 P_{G_{k}^{\max }(l)} P_{G_{j}^{\max }(l)} \mathcal{E}_{G_{k}^{\max }(l)} \\
& +\frac{\sum \sum Q_{i}\left(\left|Q_{l}\right|-2\right)}{\gamma\left(\left|Q_{l}\right|-1\right)^{2}\left(\mid Q_{i}\right)},
\end{aligned}
$$$$
\sum_{G_{i} \in Q_{l}} P_{-G_{i}^{\max }(l)}^{2} \frac{x_{G_{i}}(l)}{\gamma}=
$$$$
\frac{\sum \sum \sum_{\begin{array}{c}
G_{i}, G_{j}, G_{k} \in Q_{l} \\
G_{i} \neq G_{j} \neq G_{k}
\end{array}} \sum_{\begin{array}{c}
G_{r} \in Q_{l} \\
G_{r} \neq G_{i}, G_{j}, G_{k}
\end{array}} x_{G_{j}}(l) P_{G_{r}^{\max }(l)}}{\gamma\left(\left|Q_{l}\right|-1\right)^{2}\left(\left|Q_{l}\right|-3\right)}
$$$$
+\frac{\sum \sum \sum_{\substack{G_{i}, G_{j}, G_{k} \in Q_{l} \\ G_{i} \neq G_{j} \neq G_{k}}} x_{G_{j}}(l) P_{G_{k}^{\max }(l)}}{\gamma\left(\left|Q_{l}\right|-1\right)^{2}\left(\left|Q_{l}\right|-2\right)} .
$$

Using Eq. (21) and (41) - (42) we obtain

$$
\begin{aligned}
& \sum_{G_{i} \in Q_{l}}\left[\bar{\Phi}_{G_{i}^{\max }(l)}^{2}-2 P_{-G_{i} \max (l)} \bar{\Phi}_{G_{i}^{\max }(l)}\left(\frac{\mathcal{E}_{-G_{i} \max (l)}+x_{G_{i}}(l)}{\gamma}\right)\right] \\
& +\sum_{G_{i} \in Q_{l}} \Gamma_{G_{i}}^{l}=0 .
\end{aligned}
$$

Consequently, it is sufficient to find a $m_{\left(j, G_{i}\right)} \in \mathcal{M}_{i}$ so that $V_{\left(j, G_{i}\right)}\left(m_{-\left(j, G_{i}\right)}^{*}, m_{\left(j, G_{i}\right)}\right) \geq 0$. Set $x_{\left(j, G_{i}\right)}$ equal to 0 . We separately examine different cases, as follows.

- If $x_{G_{i}}^{*}(l)>0$ then, $\left.t_{\left(j, G_{i}\right)}^{l}\right|_{\left(m_{-\left(j, G_{i}\right)}^{*}, m_{\left(j, G_{i}\right)}\right)}=0$ because $j \notin G_{i}{ }^{\max }(l)$.

- If $x_{G_{i}}^{*}(l)=0$, then in accordance to the possible cases we define,

$$
\pi_{\left(j, G_{i}\right)}^{l}:= \begin{cases}\pi_{\left(j, G_{i}\right)}^{* l}, & \text { for Case B, Part BI; } \\ P_{G^{\max }(l)}^{*}, & \text { for Case B, Part BII; } \\ \pi_{\left(j, G_{i}\right)}^{* l}, & \text { for Case C, Part CI; } \\ P_{G^{\max }(l)}^{*}, & \text { for Case C, Part CII; } \\ \varpi_{D I}^{*}(l), & \text { for Case D, Part DI; } \\ \varpi_{D I I}^{*}(l), & \text { for Case D, Part DII. }\end{cases}
$$

where, $\varpi_{D I}^{*}(l):=$

$$
\frac{\frac{1}{\left|G_{i}^{\max }(l)\right|}\left[P_{G^{\max }(l)}^{*}-\sum_{\substack{j \in G_{i}^{\max } \\ j \neq i}} \pi_{j, G_{i}^{\max }}^{*}+\frac{\mathcal{E}_{-G_{i} \max (l)}^{*}}{\gamma}\right.}{\sqrt{\left.\left[P_{G^{\max }(l)}^{*} \frac{\mathcal{E}_{-G_{i} \max (l)}^{*}}{\gamma}\right]^{2}+\frac{P_{G^{\max (l)}}^{*} \sum \sum_{G_{j}, G_{j} \in Q_{l}} x_{G_{j}}^{*}(l)}{\left|Q_{l}\right|-1}\right]}}
$$

Next we consider all links $l \in \mathbf{L}$ where $\left|Q_{l}\right| \leq 3$; let these link be $l_{1}, l_{2}, \cdots, l_{r}$. Then, by using the above equality and the specification of the tax function for the links $l_{1}, l_{2}, \cdots, l_{r}$ (cf. section III, cases B and C) we obtain

$$
\begin{aligned}
\sum_{\left(j, G_{i}\right) \in \cup_{G_{i} \in \mathcal{N}} G_{i}} t_{\left(j, G_{i}\right)} & \sum_{l \in \mathbf{L}} \sum_{G_{i} \in Q_{l}} \sum_{\left(j, G_{i}\right) \in G_{i}(l)} t_{\left(j, G_{i}\right)}^{l} \\
= & \sum_{l \in \mathbf{L}:\left|Q_{l}\right|=2} \sum_{G_{i} \in Q_{l}} \sum_{\left(j, G_{i}\right) \in G_{i} \max (l)} t_{\left(j, G_{i}\right)}^{l} \\
& +\sum_{l \in \mathbf{L}:\left|Q_{l}\right|=3} \sum_{G_{i} \in Q_{l}} \sum_{\left(j, G_{i}\right) \in G_{i} \max (l)}^{t_{\left(j, G_{i}\right)}^{l}} \sum_{l \in \mathbf{L}:\left|Q_{l}\right|>3} \sum_{G_{i} \in Q_{l}} \sum_{\left(j, G_{i}\right) \in G_{i} \max (l)} t_{\left(j, G_{i}\right)}^{l}+\sum_{j=1}^{r} \mathcal{S}^{l_{j}} \\
& +\sum
\end{aligned}
$$

The last equality in (44) is true for the following reason. By Eq. (43) the third sum on the right hand side of the second equality in (44) is equal to zero. The sum of the three remaining terms is also equal to zero because of Eqs. (10)(25).

Proof of Theorem 5: We need to show that

$V_{\left(j, G_{i}\right)}\left(m^{*}\right)=\left[U_{\left(j, G_{i}\right)}\left(x_{\left(j, G_{i}\right)}\right)-\sum_{l \in \mathcal{R}_{\left(j, G_{i}\right)}} t_{\left(j, G_{i}\right)}^{l}\right]_{m=m^{*}} \geq 0$,

for every $\left(j, G_{i}\right), G_{i} \in \mathcal{N}$. By the property of NE, it follows that

$$
V_{\left(j, G_{i}\right)}\left(m^{*}\right) \geq V_{\left(j, G_{i}\right)}\left(m_{-\left(j, G_{i}\right)}^{*}, m_{\left(j, G_{i}\right)}\right) .
$$

and

$$
\begin{aligned}
& \varpi_{D I I}^{*}(l):=P_{G^{\max }(l)}^{*}\left[1+\frac{\mathcal{E}_{-G_{i} \max (l)}^{*}}{\gamma}\right] \\
& +\sqrt{\left[P_{G^{\max }(l)}^{*} \frac{\mathcal{E}_{-G_{i} \max (l)}^{*}}{\gamma}\right]^{2}+\frac{P_{G^{\max }(l)}^{*} \sum_{G_{j}, G_{j} \in Q_{l}} x_{G_{j}}^{*} \neq G_{i}}{\left|Q_{l}\right|-1}} .
\end{aligned}
$$

We can ${ }^{7}$ show that $t_{\left(j, G_{i}\right)}^{l}$ for every $l \in \mathcal{R}_{\left(j, G_{i}\right)}$ is equal to zero at

$m_{\left(j, G_{i}\right)}=\left(0, \pi_{\left(j, G_{i}\right)}^{l_{1}}, \cdots, \pi_{\left(j, G_{i}\right)}^{l_{\left|\mathcal{R}_{\left(j, G_{i}\right)}\right|}}\right)$ when $\pi_{\left(j, G_{i}\right)}^{l_{k}}, 1 \leq k \leq$ $\left|\mathcal{R}_{\left(j, G_{i}\right)}\right|$ is defined by (46).

In the other hand, by $m_{\left(j, G_{i}\right)}$ where its arguments are defined in the above, we obtain

$$
\begin{array}{r}
V_{\left(j, G_{i}\right)}\left(m_{-\left(j, G_{i}\right)}^{*}, m_{\left(j, G_{i}\right)}\right)= \\
U_{\left(j, G_{i}\right)}(0)-\sum_{l \in \mathcal{R}_{\left(j, G_{i}\right)}} t_{\left(j, G_{i}\right)}^{l}\left(m_{-\left(j, G_{i}\right)}^{*}, m_{\left(j, G_{i}\right)}\right) \\
=U_{\left(j, G_{i}\right)}(0)=0,
\end{array}
$$

when $\left(j, G_{i}\right) \neq k_{l_{1}}, k_{l_{2}}, \cdots, k_{l_{r}}$.

When $\left(j, G_{i}\right)=k_{l_{q}}, q=1,2, \cdots, r$,

$V_{\left(j, G_{i}\right)}\left(m_{-\left(j, G_{i}\right)}^{*}, m_{\left(j, G_{i}\right)}\right)=$

$$
\begin{aligned}
U_{\left(j, G_{i}\right)}(0) & -\sum_{l \in \mathcal{R}_{\left(j, G_{i}\right)}} t_{\left(j, G_{i}\right)}^{l}\left(m_{-\left(j, G_{i}\right)}^{*}, m_{\left(j, G_{i}\right)}\right)-\mathcal{S}^{* l_{q}} \\
& =-\mathcal{S}^{* l_{q}} \geq 0
\end{aligned}
$$

\footnotetext{
${ }^{7}$ Since $\gamma$ is sufficiently large then it is guaranteed that $\varpi_{D I}$ and $\varpi_{D I I}$ are positive.
} 
Combining (45), (47) and (48) we obtain

$V_{\left(j, G_{i}\right)}\left(x_{\left(j, G_{i}\right)}^{*}, t^{*}\right) \geq\left. V_{\left(j, G_{i}\right)}(x, t)\right|_{m=\left(m_{\left(j, G_{i}\right)}, m_{-\left(j, G_{i}\right)}^{*}\right)} \geq 0$

Proof of Theorem 6: Let $m^{*}$ be an arbitrary $N E$ of the game $(\mathcal{M}, f, V)$ induced by the proposed game form. Consider problem Max.1, since the functions $U_{\left(j, G_{i}\right)}, j \in$ $G_{i}, G_{i} \in \mathcal{N}$, are concave and differentiable and the constraints are linear, Slater's condition ( [25]) is satisfied, the duality gap is equal to zero, and Karush Kuhn Tucker (KKT) conditions are necessary and sufficient to guarantee the optimality of any allocation $x$ that satisfies them. Let $\lambda^{l}$ be the Lagrange multiplier corresponding to the capacity constraint for link $l$ and $\nu_{i}$ be the Lagrange multiplier corresponding to the demand constraint. The Lagrangian for problem Max.1 is

$\mathcal{L}(x, \lambda, \nu)=$

$$
\begin{aligned}
& \sum_{G_{i} \in \mathcal{N}} \sum_{\left(j, G_{i}\right) \in G_{i}} U_{\left(j, G_{i}\right)}\left(x_{\left(j, G_{i}\right)}\right) \\
& -\sum_{l \in \mathbf{L}} \sum_{e(l) \in E(l)} \lambda_{e(l)}\left[\sum_{G_{i} \in Q_{l}} x_{\left(j, G_{i}\right)} \mathbb{I}\left\{\left(j, G_{i}\right) \in G_{i}(l)\right\}-c_{l}\right] \\
& +\sum_{G_{i} \in \mathcal{N}} \sum_{\left(j, G_{i}\right) \in G_{i}} \nu_{\left(j, G_{i}\right)} x_{\left(j, G_{i}\right)}
\end{aligned}
$$

and the KKT conditions are:

$$
\begin{aligned}
& \frac{\partial \mathcal{L}\left(x^{*}, \lambda^{*}, \nu^{*}\right)}{\partial x_{\left(j, G_{i}\right)}}=\frac{\partial U_{\left(j, G_{i}\right)}\left(x_{\left(j, G_{i}\right)}^{*}\right)}{\partial x_{\left(j, G_{i}\right)}}+\nu_{\left(j, G_{i}\right)}^{*} \lambda_{e\left(l,\left(j, G_{i}\right)\right)}^{*}=0 \\
& \quad-\sum_{l \in \mathcal{R}_{\left(j, G_{i}\right)}} \sum_{e\left(l,\left(j, G_{i}\right)\right) \in E\left(l,\left(j, G_{i}\right)\right)} \\
& \lambda_{e(l)}^{*}\left[\sum_{G_{i} \in Q_{l}} x_{\left(j, G_{i}\right)}^{*} \mathbb{I}\left\{\left(j, G_{i}\right) \in G_{i}(l)\right\}-c_{l}\right]=0, \forall l \\
& \nu_{\left(j, G_{i}\right)}^{*} x_{\left(j, G_{i}\right)}^{*}=0 \quad \forall G_{i} \in \mathcal{N} \text { and } j \in G_{i} .
\end{aligned}
$$

Now, define $\forall l \in \mathbf{L}, G_{i} \in Q_{l}, j \in G_{i}{ }^{\max }(l)$

$$
\lambda_{\left(j, G_{i}\right)}^{* l}:=\sum_{e^{\max }\left(l,\left(j, G_{i}\right)\right) \in E^{\max }\left(l,\left(j, G_{i}\right)\right)} \lambda_{e\left(l,\left(j, G_{i}\right)\right)}^{*},
$$

where, $E^{\max }(l)$ is a subset of equations, $e^{\max }(l)$ of (5), such that every element $x_{\left(k, G_{s}\right)} \in e^{\max }(l)$ is equal to $x_{G_{s}}(l)$, and accordingly, we can define $E^{\max }\left(l,\left(j, G_{i}\right)\right)$ and $e^{\max }\left(l,\left(j, G_{i}\right)\right)$.

Furthermore, (51) implies the following, $\forall l \in \mathbf{L}$ and $G_{i} \in$ $Q_{l}, j \in G_{i}(l)$,

$$
\text { if } x_{\left(j, G_{i}\right)}<x_{G_{i}}(l) \text { then } \lambda_{e\left(l,\left(j, G_{j}\right)\right)}^{*}=0 .
$$

Since $m^{*}$ is a NE then for every user $\left(j, G_{i}\right), G_{i} \in \mathcal{N}, j \in G_{i}$, there exists at least a link in $\mathcal{R}_{\left(j, G_{i}\right)}$ such that $x_{\left(j, G_{i}\right)}=$ $x_{G_{i}}(l)$. Now, by using (53) and (54) we can reformulate the KKT constraints as follows, suppose that at link $l \in$ $\mathcal{R}_{\left(j, G_{i}\right)}, x_{\left(j, G_{i}\right)}=x_{G_{i}}(l)$, then

$$
\begin{aligned}
& \frac{\partial \mathcal{L}\left(x^{*}, \lambda^{*}, \nu^{*}\right)}{\partial x_{G_{i}}^{*}(l)}=\frac{\partial U_{\left(j, G_{i}\right)}\left(x_{G_{i}}^{*}(l)\right)}{\partial x_{G_{i}}(l)} \\
& \quad-\sum_{l \in \mathcal{R}_{\left(j, G_{i}\right)}^{\max }} \lambda_{\left(j, G_{i}\right)}^{* l}+\nu_{\left(j, G_{i}\right)}^{*}=0 \\
& \lambda^{* l}\left[\sum_{G_{i} \in Q_{l}} x_{G_{i}}^{*}(l)-c_{l}\right]=0, \quad \forall l \in \mathbf{L} \\
& \nu_{\left(j, G_{i}\right)}^{*} x_{G_{i}}^{*}(l)=0 \quad \forall G_{i} \in \mathcal{N} \text { and } j \in G_{i} .
\end{aligned}
$$

where $\lambda^{* l}:=\sum_{j \in G_{i} \max (l)} \lambda_{\left(j, G_{i}\right)}^{* l}$ for every $G_{i} \in Q_{l}$. Because of the characteristics of problem Max.1, KKT conditions are necessary and sufficient for any optimal solution of Max.1. Therefore, to show that any arbitrary NE $m^{*}$ of the specified game, induced from the game form presented in section III, is correspondent to an optimal solution, it is enough to find $\nu_{i}^{*}$, $\lambda^{l *}$, and $\lambda_{\left(j, G_{i}\right)}^{* l}$, for every $G_{i} \in \mathcal{N}, j \in G_{i}, l \in \mathbf{L}$, appropriately, such that Eqs. (55), (56) and (57) are satisfied. If we set $\nu_{\left(j, G_{i}\right)}^{*}, G_{i} \in \mathcal{N}, j \in G_{i}$, equal to zero, then (57) is satisfied. In addition, if we set $\lambda^{l *}=P_{G^{\max }(l)}^{*}, l \in \mathbf{L}$ and $\lambda_{\left(j, G_{i}\right)}^{* l}$ equal to (28), then the correctness of (56) and (57) will be implied from (27) and (28), respectively. Furthermore, by the construction of the game form $\sum_{G_{i} \in \mathcal{N}} \sum_{j \in G_{i}} \sum_{l \in \mathcal{R}_{\left(j, G_{i}\right)}} t_{\left(j, G_{i}\right)}^{* l}$ is equal to zero. Consequently, the NE $m^{*}$ results in an optimal solution of problem Max.0. Since the NE $m^{*}$ was arbitrary chosen, every NE $m^{*}$ of the game induced by the game form proposed in section III results in an optimal solution of problem Max.0. 